\title{
Electronic structure of bulk manganese oxide and nickel oxide from coupled cluster theory
}

\author{
Yang Gao $\odot,{ }^{1}$ Qiming Sun, ${ }^{2,3}$ Jason M. Yu $\odot,{ }^{3,4}$ Mario Motta $\odot,{ }^{5,3}$ James McClain, ${ }^{3}$ Alec F. White $\odot,{ }^{3}$ \\ Austin J. Minnich, ${ }^{1, *}$ and Garnet Kin-Lic Chan ${ }^{3, \dagger}$ \\ ${ }^{1}$ Division of Engineering and Applied Science, California Institute of Technology, Pasadena, California 91125, USA \\ ${ }^{2}$ AxiomQuant Investment Management LLC, Shanghai 200120, China \\ ${ }^{3}$ Division of Chemistry and Chemical Engineering, California Institute of Technology, Pasadena, California 91125, USA \\ ${ }^{4}$ Department of Chemistry, University of California, Irvine, California 92521, USA \\ ${ }^{5}$ IBM Almaden Research Center, San Jose, California 95120, USA
}

(Received 13 December 2019; accepted 27 March 2020; published 27 April 2020)

\begin{abstract}
We describe the ground- and excited-state electronic structure of bulk $\mathrm{MnO}$ and $\mathrm{NiO}$, two prototypical correlated electron materials, using coupled cluster theory with single and double excitations (CCSD). As a corollary, this work also reports an implementation of unrestricted periodic $a b$ initio equation-of-motion CCSD. Starting from a Hartree-Fock reference, we find fundamental gaps of 3.46 and $4.83 \mathrm{eV}$ for $\mathrm{MnO}$ and $\mathrm{NiO}$, respectively, for the 16-unit supercell, slightly overestimated compared to experiment, although finite-size scaling suggests that the gap is more severely overestimated in the thermodynamic limit. From the character of the correlated electronic bands we find both $\mathrm{MnO}$ and $\mathrm{NiO}$ to lie in the intermediate Mott/charge-transfer insulator regime, although $\mathrm{NiO}$ appears as a charge transfer insulator when only the fundamental gap is considered. While the lowest quasiparticle excitations are of metal $3 d$ and $\mathrm{O} 2 p$ character in most of the Brillouin zone, near the $\Gamma$ point, the lowest conduction band quasiparticles are of $s$ character. Our study supports the potential of coupled cluster theory to provide high-level many-body insights into correlated solids.
\end{abstract}

DOI: 10.1103/PhysRevB.101.165138

\section{INTRODUCTION}

Understanding the properties of solids with correlated electrons is a long-standing challenge. The first-row transitionmetal oxides with partially filled $d$ shells, such as $\mathrm{MnO}$ and $\mathrm{NiO}$, are prominent examples. The partially filled $d$ band suggests that these materials should be metals. However, experimentally, they are found to be insulators with large gaps [1-3]. One of the first proposed explanations for this discrepancy was that the gap originates from electron interactions, forming a so-called Mott insulator [4]. Later work, however, based on correlating model cluster calculations to observed spectra [1,5], suggested that the gap corresponds to a ligandto-metal charge-transfer process. Since then, the character of the insulating state across the first-row transition-metal oxides has been a fertile topic of study [6].

In principle, these questions could be unambiguously resolved through accurate first-principles calculation on the bulk material. However, achieving quantitative accuracy for the properties of greatest interest for transition-metal oxides has been difficult. For example, local and gradient density functional theories (DFTs) typically underestimate both the insulating gap and order parameters, such as the magnetic moment [7]. While hybrid functionals can give better gaps, this success does not always translate to better properties and is not consistent across materials [8-10]. Quantum Monte Carlo methods

\footnotetext{
*aminnich@ caltech.edu

†gkc1000@gmail.com
}

can provide greater accuracy at higher cost $[11,12]$ but do not allow access to the full spectrum. Low-order diagrammatic approaches such as the GW approximation [13-16] have also been applied to these systems, with mixed success. Finally, while DFT with a Hubbard $U(\mathrm{DFT}+U)$ [17-19] and dynamical mean-field theory (DMFT) calculations [20-28] have provided a practical approach to obtain important insights, these methods contain a degree of empiricism that introduces uncertainty into the interpretations.

Coupled cluster (CC) theory is a theoretical framework originating in quantum chemistry and nuclear physics [29-31], which has recently emerged as a new way to treat electronic structure in solids at the many-body level [32,33]. The method is systematically improvable in terms of particlehole excitation levels, giving rise to the coupled cluster with singles, doubles, triples, and higher approximations. While the earliest formulation was for ground states, excited states can be computed via the equation-of-motion (EOM) formalism [29,34-36]. Recent single-particle spectra computed for the electron gas [37] and simple covalent solids [32] demonstrate that high accuracies can be achieved at the level of coupled cluster singles and doubles (CCSD). Note that at the ground-state CCSD level, the coupled cluster energy includes all ladders and ring diagrams, some of the couplings between the two, and partially self-consistently renormalized propagators. Thus compared to approximate GW methods, the CCSD equations are less sensitive to the single-particle starting point, while the inclusion of ladders (which are entirely omitted in $\mathrm{GW}$ ) provides for some ability to treat stronger correlations. A detailed comparison of the diagrammatic content of GW and excited-state EOM-CCSD can be found in [38]. 
In this work, we use coupled cluster theory, in its singles and doubles approximation (CCSD and EOM-CCSD), to describe the ground and excited states of the prototypical transition-metal oxides, $\mathrm{MnO}$ and $\mathrm{NiO}$. Since the ground states are magnetically ordered, we use the unrestricted CC formalism, and our work also reports the implementation of unrestricted EOM-CCSD in a periodic system. Our largest calculations treat a $2 \times 2 \times 2$ supercell of the antiferromagnetic unit cell, with 16 metals and 16 oxygens, correlating up to 384 electrons. We analyze both the ground state and the correlated single-particle spectra to report on the character of the insulating state of $\mathrm{MnO}$ and $\mathrm{NiO}$. While such systems have been studied intensely with a variety of many-body methods, including several $G W$ approximations [13-16,39], dynamical mean-field theory [20-24,26,27], and quantum Monte Carlo methods $[11,12]$, the treatment by coupled cluster theory provides an independent high-level benchmark for many properties with a technique where both local and nonlocal interactions are treated on the same footing and, further, as we shall see, yields insights into the physics of these materials.

The rest of the paper is organized as follows. In Sec. II we recapitulate the ground-state coupled cluster theory and EOM formalism for excited states in periodic systems. In Sec. III we present the CCSD calculations for $\mathrm{NiO}$ and $\mathrm{MnO}$, together with an analysis of the numerical convergence and character of the states. We finish with some conclusions in Sec. IV.

\section{METHODS}

\section{A. Periodic ground-state coupled cluster theory}

In the coupled cluster formalism, the ground state is described using an exponential excitation ansatz,

$$
\left|\Psi_{0}\right\rangle=e^{\hat{T}}|0\rangle
$$

where $|0\rangle$ is a single-determinant reference state obtained from a mean-field theory such as the Hartree-Fock theory or Kohn-Sham density functional theory and the excitation operator $\hat{T}$ is a sum over single, double, triple, and higher (particle-hole) excitations,

$$
\hat{T}=\sum_{\mu} \hat{t}_{\mu} .
$$

Here $\hat{t}_{\mu}$ creates a linear combination of $\mu$-particle- $\mu$-hole excitations). In CCSD, the cluster operator is truncated at the singles and doubles level so that $\hat{T}=\hat{t}_{1}+\hat{t}_{2}$. In a system with crystal translational symmetry, the particle and hole states carry a crystal momentum label; thus

$$
\begin{gathered}
\hat{t}_{1}=\sum_{k_{i}}^{\prime} \sum_{i a} t_{i k_{i}}^{a k_{a}} \hat{E}_{i k_{i}}^{a k_{a}}, \\
\hat{t}_{2}=\frac{1}{4} \sum_{k_{i} k_{a} k_{j} k_{b}}^{\prime} \sum_{i a j b} t_{i k_{i} j k_{j}}^{a k_{b} b k_{b}} \hat{a}_{a k_{a}}^{\dagger} \hat{a}_{b k_{b}}^{\dagger} \hat{a}_{i k_{i}} \hat{a}_{j k_{j}},
\end{gathered}
$$

where $i, j, k, l, \ldots$ denote occupied (hole) spin-orbital labels, $a, b, c, d, \ldots$ denote virtual (particle) labels, $k_{i}, k_{j}, \ldots$ denote crystal momenta, and $\hat{E}_{i k_{i}}^{a k_{a}}=\hat{a}_{a k_{a}}^{\dagger} \hat{a}_{i k_{i}}$. The primed sum indicates crystal momentum conservation, i.e., $k_{a}+k_{b}-k_{i}-$ $k_{j}=G$, where $G$ is a reciprocal lattice vector. The excitation amplitudes and ground-state energy $E$ are obtained by solving the Schrödinger equation for the similarity transformed Hamiltonian $\bar{H}[29,30]$,

$$
\begin{gathered}
\bar{H}=e^{-\hat{T}} \hat{H} e^{\hat{T}}, \\
E=\langle 0|\bar{H}| 0\rangle, \\
0=\langle\mu|\bar{H}| 0\rangle \quad \forall \mu .
\end{gathered}
$$

In this work, because of the spin ordering in the ground states of $\mathrm{MnO}$ and $\mathrm{NiO}$, we will use the unrestricted form of the coupled cluster theory. Here $|0\rangle$ is a broken-symmetry mean-field state (an eigenfunction of $\hat{S}_{z}$ but not necessarily $\hat{S}^{2}$ as, for example, in an antiferromagnetic state), and $\hat{t}_{1}$ and $\hat{t}_{2}$ preserve $S_{z}$. The detailed periodic unrestricted CC groundstate equations are given in the Appendix as an extension of the molecular equations in Refs. [40,41].

\section{B. Periodic equation-of-motion coupled cluster theory}

Excited-state wave functions and energies in $\mathrm{CC}$ can be obtained through the EOM formalism. This amounts to diagonalizing the similarity transformed Hamiltonian $\bar{H}$ in the excitation space of interest [29,30,36,41]. In this work, we compute single-particle spectra. At the EOM-CCSD level of approximation, we obtain the ionized $[N-1$; ionization potential (IP)] states by diagonalizing in the space of one-hole (1h) and two-hole, one-particle (2h1p) states, while we compute the electron attached $(\mathrm{EA} ; N+1)$ states in the space of one-particle (1p) and two-particle, one-hole ( $2 \mathrm{p} 1 \mathrm{~h})$ states. The $n$th excited-state wave function with momentum $k\left|\Psi_{n, k}^{N \pm 1}\right\rangle$ is constructed as

$$
\begin{gathered}
\left|\Psi_{n, k}^{N \pm 1}\right\rangle=\hat{R}_{n, k}^{ \pm}\left|\Psi_{0}\right\rangle, \\
\hat{R}_{n, k}^{-}=\sum_{i} r_{i k} \hat{a}_{i k}+\frac{1}{2} \sum_{k_{b} k_{i} k_{j}}^{\prime} \sum_{b i j} r_{i k_{i} j k_{j}}^{b k_{b}} \hat{E}_{j k_{j}}^{b k_{b}} \hat{a}_{i k_{i}}, \\
\hat{R}_{n, k}^{+}=\sum_{a} r^{a k} \hat{a}_{a k}^{\dagger}+\frac{1}{2} \sum_{k_{a} k_{b} k_{j}}^{\prime} \sum_{a b j} r_{i k_{i}}^{a k_{a} b k_{b}} \hat{a}_{a k_{a}}^{\dagger} \hat{E}_{j k_{j}}^{b k_{b}} .
\end{gathered}
$$

(The $n$ index has been omitted from the right-hand side for clarity; the distinction between subscripts and superscripts is not material but has been made for correspondence with the literature.) In this work, we use unrestricted $\hat{R}$ operators, where $\hat{R}^{+}$and $\hat{R}^{-}$raise and lower $S_{z}$ by $1 / 2$, respectively. In the diagonalization step, the dominant computational operation is the multiplication of $\bar{H}$ onto the vector of the $R$ amplitudes. The periodic unrestricted equation-of-motion $\mathrm{CC}$ formalism has not previously been reported, and detailed equations are given in the Appendix.

\section{Computational details}

$\mathrm{MnO}$ and $\mathrm{NiO}$ both crystallize in a rocksalt structure. Below the Néel temperature, the electrons are spin polarized in stacked ferromagnetic (111) planes with alternating spin orientations along the [111] direction, as shown in Fig. 1. In our calculations, we used supercells that are multiples of a rhombohedral unit cell with four atoms to host the antiferromagnetic (AFM) order. All calculations were performed with 


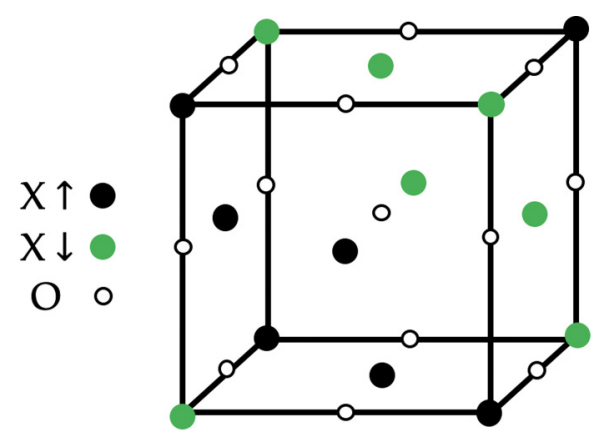

FIG. 1. The cell structure of transition-metal monoxide $X \mathrm{O}(X=$ $\mathrm{Mn}, \mathrm{Ni}$ ) in the AFM II phase. The black and green solid circles denote metal atoms with opposite spin orientations, and the open circles denote the oxygens.

the experimental lattice constants at $300 \mathrm{~K}$, i.e., $a=4.43 \AA$ and $a=4.17 \AA$ for $\mathrm{MnO}$ and $\mathrm{NiO}$, respectively [42].

We used the Goedecker-Teter-Hutter (GTH) pseudopotential and corresponding single-particle basis [43] downloaded from the CP2K package [44]. Most of our calculations used the GTH-DZVP-MOLOPT-SR basis, with 78 orbitals per rhombohedral unit cell; we carried out a subset of calculations using the GTH-SZV/DZVP/TZVP-MOLOPT(-SR) (SZV/DZVP/TZVP for short) for the metal and oxygen, respectively [43], to assess basis set convergence. Electron repulsion integrals were generated by periodic Gaussian density fitting with an even-tempered Gaussian auxiliary basis [45]. The initial reference state for the $\mathrm{CC}$ calculations was generated from unrestricted Hartree-Fock (UHF) and Kohn-Sham density functional calculations. Ground-state unrestricted CCSD energies and observables used a $2 \times 2 \times 2$ Monkhorst-Pack $k$ mesh together with a $2 \times 2 \times 2$ twist average [46,47] to effectively obtain a $4 \times 4 \times 4$ sampling of the Brillouin zone. The $\mathrm{CC}$ reduced density matrices were computed using only the right eigenvector of $\bar{H}$ [48]. Atomic character analysis and local magnetic moments on the metals were computed by population analysis in the crystalline intrinsic atomic orbital basis [49,50]. Equation-of-motion CCSD band structures were generated using the IP/EA energies at the twisted supercell reciprocal lattice origin. We sampled these twists along the high-symmetry lines defined by the underlying nonmagnetic primitive cell (as generally reported in experiments [51]). Note that many theoretical calculations report magnetic symmetry labels associated with the four-atom rhombohedral cell, and we additionally use such labels in parentheses when the high-symmetry points of the primitive and rhombohedral cells coincide. All methods were implemented within and calculations were performed using the PYSCF package [52].

\section{RESULTS}

\section{A. Numerical convergence and $\mathrm{CC}$ orbital dependence}

The CC calculations for these materials are computationally very demanding, and it is not possible to simply calculate properties at the basis set and thermodynamic limit. We thus first roughly assess the convergence of our numerical results. In Table I we report the CC total energy (starting from UHF orbitals), Ni and Mn magnetic moments,
TABLE I. Basis set convergence of CCSD total energy, metal magnetic moment, and direct $\Gamma$ gap $\Delta_{\Gamma}$ and indirect fundamental gap $\Delta_{\text {ind }}$ for a $1 \times 1 \times 1$ cell.

\begin{tabular}{llcccc}
\hline \hline System & Basis & $E_{\mathrm{CC}}(\mathrm{eV})$ & $\mu_{B}$ & $\Delta_{\Gamma}^{\mathrm{cc}}(\mathrm{eV})$ & $\Delta_{\text {ind }}^{\mathrm{cc}}(\mathrm{eV})$ \\
\hline $\mathrm{MnO}$ & SZV & -2.66 & 4.29 & 0.36 & 1.04 \\
& DZVP & -12.16 & 4.61 & 2.49 & 1.48 \\
& $\mathrm{TZVP}$ & -14.23 & 4.61 & 2.40 & 1.42 \\
$\mathrm{NiO}$ & $\mathrm{SZV}$ & -3.36 & 0.46 & 2.49 & 2.13 \\
& DZVP & -13.40 & 1.18 & 3.22 & 2.62 \\
& TZVP & -15.68 & 1.19 & 3.21 & 2.49 \\
\hline \hline
\end{tabular}

and the single-particle gap for a $1 \times 1 \times 1$ rhombohedral cell [both the direct gap at $\Gamma$ and the presumed fundamental gap that is an indirect transition from $\Lambda_{\frac{1}{2}}(Z)$ (the midpoint of the $\Lambda$ symmetry direction of the primitive cell, equivalent to the $Z$ high-symmetry point of the rhombohedral cell; see the $x$-axis label in Fig. 6) to $\Gamma$ ] [39]. Unless otherwise noted, the fundamental gap here will refer to this transition. As a function of increasing basis size (SZV, DZVP, TZVP), the magnetic moment is already well converged at the DZVP level, while the total energy still changes significantly, as expected. The single-particle direct gap $\Delta_{\Gamma}$ is well converged, while the indirect fundamental gap $\Delta_{\text {ind }}$ is somewhat less so, changing by more than $0.1 \mathrm{eV}$ in NiO moving from DZVP to TZVP. While the remaining basis error may be several tenths of an $\mathrm{eV}$, as a computational compromise, we use the DZVP basis for the remaining calculations.

The same quantities for a $2 \times 2 \times 2$ supercell (with twist averaging; see Sec. IIC) are shown in Table II. Both the magnetic moments and gaps change significantly from the $1 \times 1 \times 1$ cell; the change in the indirect and direct gaps ranges from 1.8 to $2.2 \mathrm{eV}$. Note that while the basis error converges the gap from above, the (larger) finite-size error converges the gap from below. We can carry out a rough finite-size scaling of the fundamental gap assuming that it scales as $N_{k}^{-\frac{1}{3}}$, where $N_{k}$ is the number of $k$ points in the mesh. This extrapolation is shown in Fig. 2 for both the UHF and CC gaps. In the thermodynamic limit (TDL), the extrapolated $\mathrm{CC}$ gaps increase by a further $\sim 2 \mathrm{eV}$ (the precise uncertainty

TABLE II. Local magnetic moment, fundamental gap, and direct $\Gamma$ gap from UHF, PBE, and CCSD with a $2 \times 2 \times 2 k$-point mesh (DZVP basis). The extrapolated TDL gap is listed in parentheses. Experimental gaps and moments are also reported (see the main text for a discussion of the comparison). The experimental magnetic moments are taken from Refs. [42,56]. The measured experimental gaps are taken from Ref. [2] for $\mathrm{MnO}$ and Ref. [1] for $\mathrm{NiO}$.

\begin{tabular}{lccccc}
\hline \hline System & Property & UHF & PBE & CCSD & Expt. \\
\hline $\mathrm{MnO}$ & $\mu_{B}$ & 4.86 & 4.56 & 4.76 & $4.58,4.79$ \\
& $\Delta_{\text {ind }}(\mathrm{eV})$ & $8.05(12.09)$ & $1.09(1.21)$ & $3.46(5.44)$ & $3.6-3.9$ \\
& $\Delta_{\Gamma}(\mathrm{eV})$ & $8.72(13.05)$ & $1.77(1.84)$ & $4.26(5.91)$ & \\
$\mathrm{NiO}$ & $\mu_{B}$ & 1.85 & 1.34 & 1.72 & $1.77,1.90$ \\
& $\Delta_{\text {ind }}(\mathrm{eV})$ & $9.51(13.95)$ & $1.19(1.38)$ & $4.83(7.04)$ & 4.3 \\
& $\Delta_{\Gamma}(\mathrm{eV})$ & $9.89(14.80)$ & $2.45(2.62)$ & $5.56(7.90)$ & \\
\hline \hline
\end{tabular}




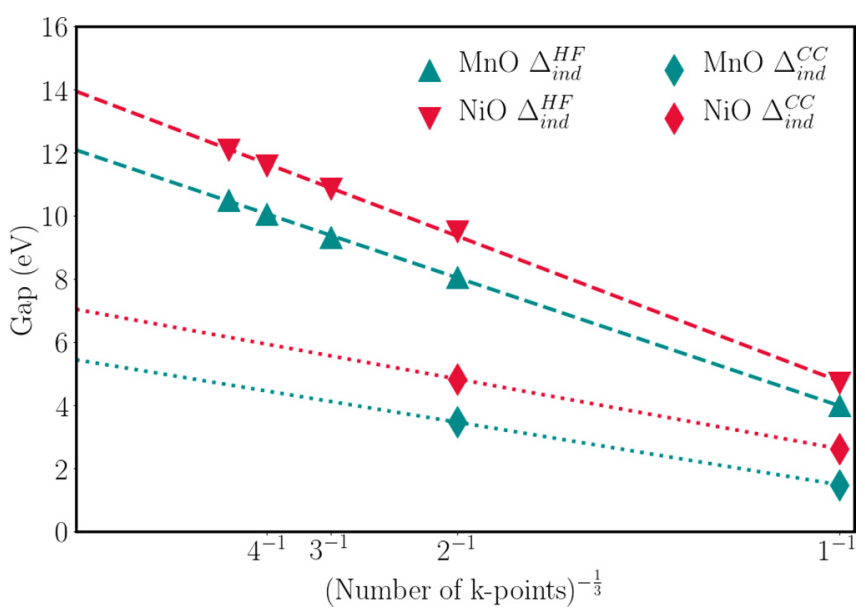

FIG. 2. Band gap extrapolation for $\mathrm{MnO}$ and $\mathrm{NiO}$. The teal and red triangles denote the $\mathrm{HF}$ indirect gap for $\mathrm{MnO}$ and $\mathrm{NiO}$, respectively. The teal and red diamonds denote the $\mathrm{CC}$ indirect gap. The dashed lines and dotted lines give the linear extrapolation to the thermodynamic limit for $\mathrm{HF}$ and $\mathrm{CC}$, respectively.

cannot be gauged from two data points). Taking into account both finite-basis-set and -size effects, the exact EOM-CCSD gaps in the TDL are thus estimated to be 1-2 eV larger than the $2 \times 2 \times 2$ results reported here.

A further variable in the $\mathrm{CC}$ calculations is the dependence on the initial mean-field orbitals. Because of the orbital relaxation generated via the $\hat{t}_{1}$ operator, this dependence is usually thought to be mild as long as one is not close to a mean-field symmetry breaking point. In Table III, we show the $\Gamma$ point direct gaps for a $1 \times 1 \times 1$ cell starting from unrestricted DFT orbitals using the local-density approximation (LDA) [53], Perdew-Burke-Ernzerhof (PBE) [54], and B3LYP [55] functionals, as well as from unrestricted HF orbitals. (Note that in $\mathrm{NiO}$, only the B3LYP density functional predicts an AFM ground state when restricted to the $1 \times 1 \times 1$ cell). For $\mathrm{MnO}$, the dependence is very weak, with a maximum variation of about $0.16 \mathrm{eV}$ in the gaps. In $\mathrm{NiO}$, the dependence is slightly larger, with a maximum variation of about $0.47 \mathrm{eV}$. This variation is smaller than the combined uncertainties due to basis and finite-size effects.

\section{B. Analysis of the ground state}

We now present a more detailed analysis of the ground states obtained by $\mathrm{CCSD}$ for $\mathrm{NiO}$ and $\mathrm{MnO}$. For all results discussed below, we used the unrestricted HF reference.

TABLE III. Direct $\Gamma$ mean-field gaps $\Delta_{\Gamma}^{\text {ref }}$ and EOM-CCSD gaps $\Delta_{\Gamma}^{\mathrm{CC}}$ starting from different mean-field orbitals for a $1 \times 1 \times 1$ cell (DZVP basis).

\begin{tabular}{clcccc}
\hline \hline System & Method & IP $(\mathrm{eV})$ & $\mathrm{EA}(\mathrm{eV})$ & $\Delta_{\Gamma}^{\text {ref }}(\mathrm{eV})$ & $\Delta_{\Gamma}^{\mathrm{CC}}(\mathrm{eV})$ \\
\hline MnO, $\Gamma$ & UHF-CC & -16.77 & -19.26 & 3.99 & 2.49 \\
& LDA-CC & -16.57 & -19.22 & 1.46 & 2.65 \\
& PBE-CC & -16.59 & -19.23 & 1.87 & 2.64 \\
& B3LYP-CC & -16.65 & -19.24 & 2.61 & 2.59 \\
\multirow{2}{*}{ NiO, $\Gamma$} & UHF-CC & -18.92 & -22.14 & 4.72 & 3.22 \\
& B3LYP-CC & -19.27 & -22.02 & 3.63 & 2.75 \\
\hline \hline
\end{tabular}
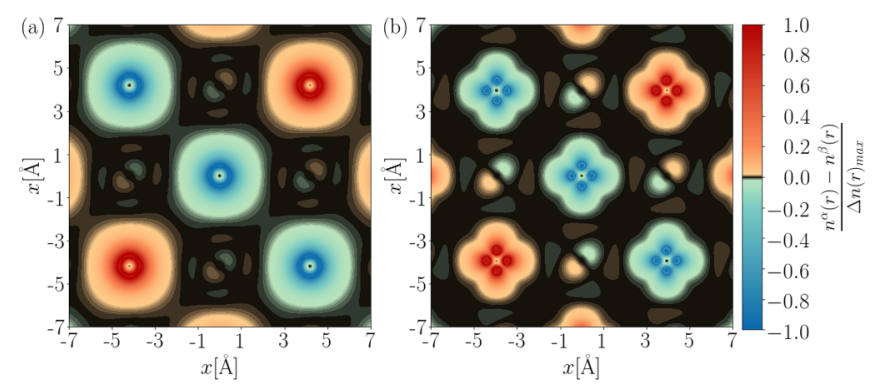

FIG. 3. Normalized spin density on the (100) surface for (a) $\mathrm{MnO}$ and (b) $\mathrm{NiO}$. The transition-metal atom is located at $(0,0)$ in the $x y$ plane. An isotropic spin density on the metal atom is observed for $\mathrm{MnO}$, and a clear $e_{g}$ symmetry is identified for $\mathrm{NiO}$.

The CC ground-state moments reported in Table II are significantly reduced from those of UHF, reflecting the wellknown observation that Hartree-Fock tends to overpolarize. Conversely, PBE underpolarizes severely in NiO. Note that the theoretical result for the magnetic moment has some variation depending on the definition of the atomic decomposition, while the experimental error bars are themselves quite large, approximately $0.2 \mu_{B}$ [57]. Thus the direct comparison between theory and experiment for this quantity should be taken with a degree of caution.

Figure 3 shows the spin density distribution of the two materials in a (100) surface cut. For $\mathrm{MnO}$, we find an isotropic spin density, as expected since all $3 d$ orbitals are partially occupied. However, for $\mathrm{NiO}$ the spin density around the $\mathrm{Ni}$ atom clearly shows $e_{g}$ occupancy, while a weakly induced spin density is also observed around the ligand oxygen site. Note that the $\mathrm{O} 2 p$ spin density is aligned in the [110] direction instead of the [100] direction, thus allowing maximal superexchange between the nearest $\mathrm{Ni}$ sites.

To further analyze the ground-state correlation, we computed the $T_{1},\left|t_{1}\right|_{\max }$, and $\left|t_{2}\right|_{\max }$ diagnostics for the CCSD wave function. These are shown in Fig. 4. The $T_{1}$ metric is the Frobenius norm (normalized by the number of correlated electrons) of the $t_{1}$ amplitudes. Previous studies have suggested that values of these diagnostics larger than $\sim 0.1$ can be considered "large" [58,59]. The $T_{1}$ and $\left|t_{1}\right|_{\max }$ metrics

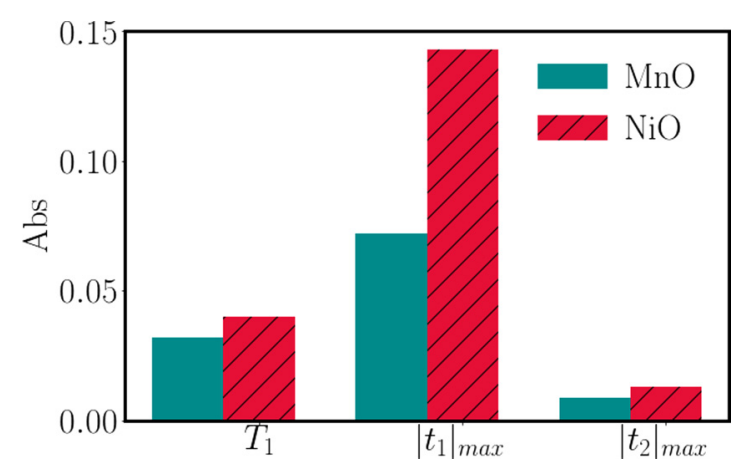

FIG. 4. CCSD amplitude diagnostics for MnO and NiO. Green columns are for $\mathrm{MnO}$, and red ones are for $\mathrm{NiO} . T_{1}$ is the Frobenius norm of the $t_{1}$ amplitudes normalized by the number of correlated electrons. $\left|t_{1}\right|_{\max }$ and $\left|t_{2}\right|_{\max }$ are the maximum value for $\left|t_{1}\right|$ and $\left|t_{2}\right|$, respectively. 


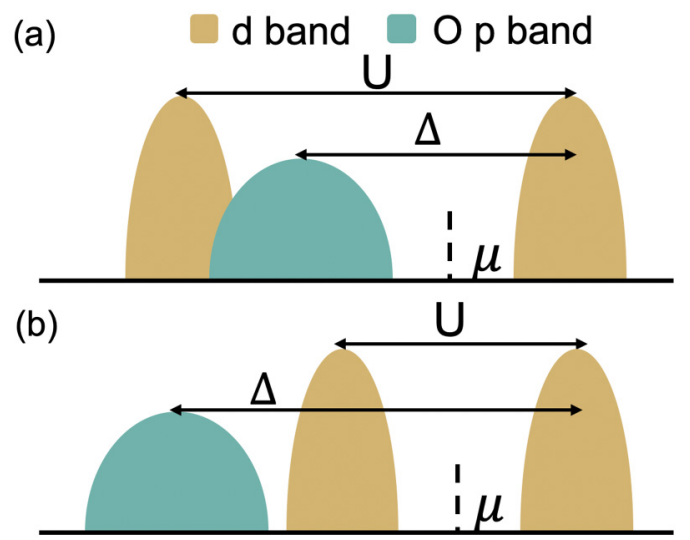

FIG. 5. Schematic diagrams of the insulating mechanism of (a) charge-transfer type, where the on-site Coulomb repulsion $U$ is smaller than the charge transfer energy $\Delta$, and (b) Mott-Hubbard type, where $\Delta>U$. Here $\mu$ is the chemical potential.

measure the importance of orbital relaxation from the meanfield reference, while $\left|t_{2}\right|_{\max }$ measures the true many-particle correlations. As seen from Fig. 4, the effect of orbital relaxation is greater in $\mathrm{NiO}$ than in $\mathrm{MnO}$, consistent with the greater degree of overpolarization of the $\mathrm{Ni}$ moment in the starting HF reference, than is seen for Mn. The small $\left|t_{2}\right|_{\max }$ values ( 0.009 for $\mathrm{MnO}$ and 0.013 for $\mathrm{NiO}$ ), however, show that both materials are reasonably described by the brokensymmetry mean-field reference.

\section{Analysis of the excited states and spectrum}

We next turn to discuss the excited states from EOMCCSD. From Table II we see that the fundamental gaps obtained by PBE and UHF for $\mathrm{MnO}$ are 1.09 and $8.05 \mathrm{eV}$, respectively, both far from the experimental estimate of 3.6$3.9 \mathrm{eV}$ [2]. In contrast, EOM-CCSD with a $2 \times 2 \times 2$ supercell finds the indirect gap to be $3.46 \mathrm{eV}$. This is similar to the 3.5-eV gap found in prior quasiparticle self-consistent $\mathrm{GW}$ (QPscGW) calculations by Faleev and coworkers [13]. In NiO we observe an indirect gap of 9.51, 1.19, and $4.83 \mathrm{eV}$ with HF, PBE, and EOM-CCSD $(2 \times 2 \times 2$ supercell), respectively. The EOM-CCSD gap is much larger than the 2.9-eV gap found by GGA-based GW [14] and close to the 4.8-eV gap found by QPscGW [13], as well as the experimental estimate of $4.3 \mathrm{eV}$ [1]. However, as discussed in Sec. III A, the estimated finite-size and basis effects in the EOM-CC calculations are quite large (TDL extrapolations in Table II are shown in parentheses); thus the final basis set limit and TDL EOM-CCSD gaps are overestimated by $1-2 \mathrm{eV}$. The sizable $T_{1}$ diagnostics in the ground state suggest that this error may arise from differential orbital relaxation between the ground and excited states. Encouragingly, however, we note that the variation in the gap due to the choice of meanfield orbitals (about $0.5 \mathrm{eV}$ ) is significantly smaller than the variation associated with different starting points in the GW approximation.

The nature of the insulating gap in $\mathrm{MnO}$ and $\mathrm{NiO}$ is of some interest. A schematic picture of the charge-transfer and Mott insulating states is shown in Fig. 5. Figure 6 plots
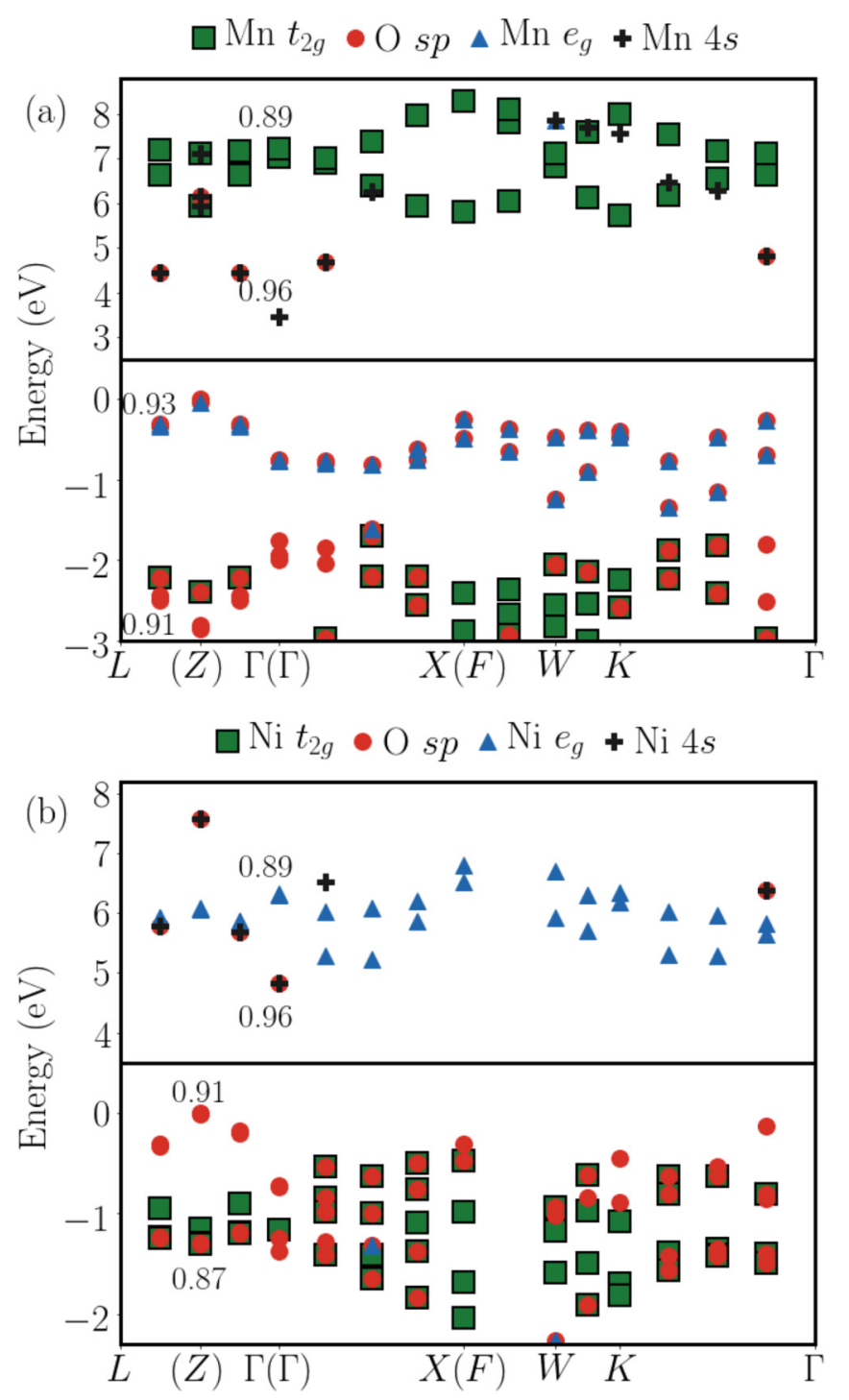

FIG. 6. Electronic structure and quasiparticle weight analysis of (a) $\mathrm{MnO}$ and (b) $\mathrm{NiO}$. The labels for the high-symmetry points are those defined by the primitive fcc cell; symmetry labels for the AFM rhombohedral cell are provided in brackets when the special points coincide. The top panel is for the conduction band, and the bottom one is for the valence band. Valence band maxima are shifted to $0 \mathrm{eV}$. Atomic character with weight larger than $30 \%$ is indicated by the symbols. Quasiparticle weights are shown for the highest and lowest roots computed at $\Gamma$ and $\Lambda_{\frac{1}{2}}(Z)$.

the correlated band structure at discrete points in reciprocal space from EOM-CC, with the atomic character labeled by the colors and symbols. Quasiparticle weights are indicated for selected excitations as the total weight of the $1 \mathrm{~h}$ (IP) or $1 \mathrm{p}$ (EA) sector, i.e., $\sum_{i}\left|r_{i k}\right|^{2}$ and $\sum_{a}\left|r^{a k}\right|^{2}$. The $k$-resolved density of states (DOS) is approximated by summing over the computed EOM-CC roots at each momentum, and the DOS at selected points in the Brillouin zone is shown in Fig. 7.

Figure 6 shows that the top of the valence band in $\mathrm{MnO}$ is hybridized between the $e_{g}$ states of $\mathrm{Mn}$ and the $\mathrm{O} 2 p$ states, while the conduction band minimum (CBM) consists 

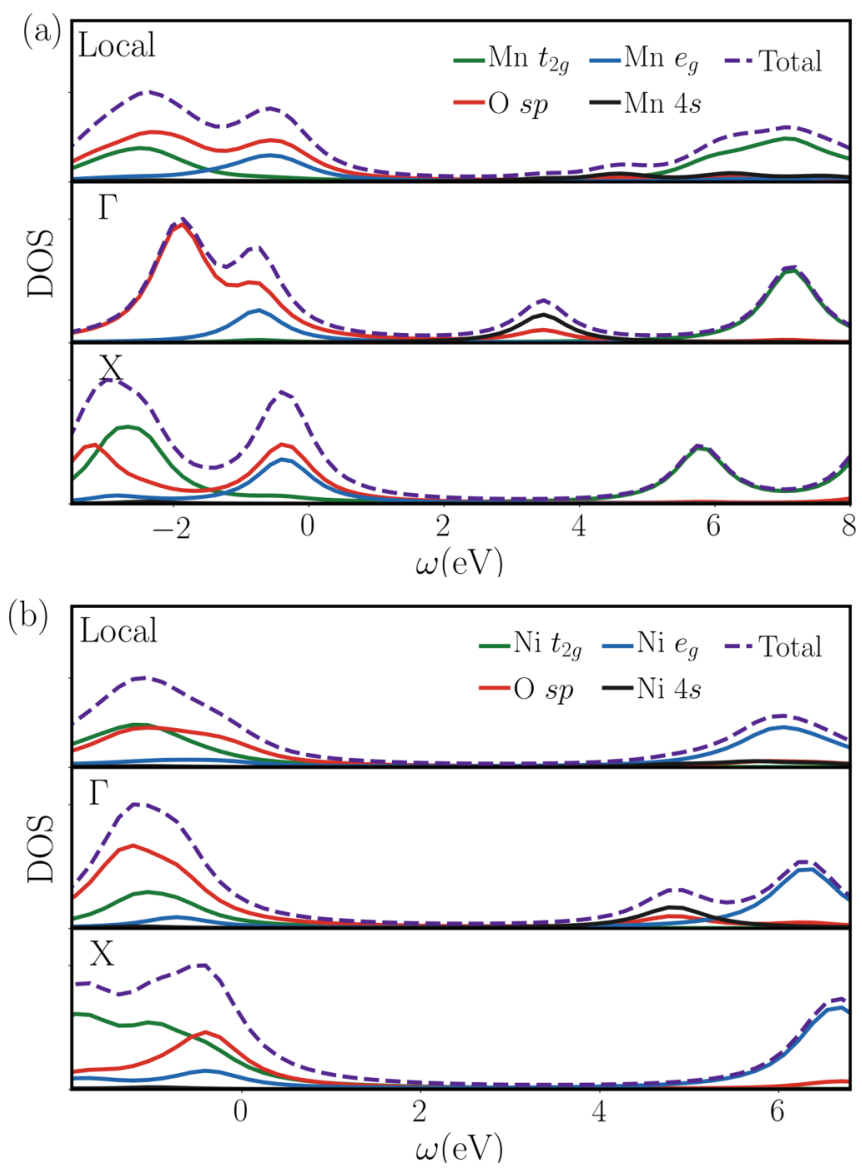

FIG. 7. Approximate density of states of (a) $\mathrm{MnO}$ and (b) $\mathrm{NiO}$ computed by summing over the EOM-CC roots. The first panel is the local DOS, and the two panels below are the DOSs at highsymmetry points $\Gamma$ and $X$. The spectral functions are computed with a Lorentzian broadening factor $\eta=0.4 \mathrm{eV}$.

mainly of nondispersive $t_{2 g}$ character, except near the $\Gamma$ point (CBM), where it has $s$ character. In $\mathrm{NiO}$, the valence band near the valence band maximum (VBM) is dominated by $\mathrm{O}$ $2 p$ states ( $81 \%$ at VBM), while the picture for the conduction band is similar to that in $\mathrm{MnO}$, including the $s$ character near the CBM. The above picture is complemented by the DOS in Fig. 7, where in $\mathrm{MnO}$, near the Fermi level, the $\mathrm{O} 2 p$ states contribute slightly more weight to the valence bands than the Mn $e_{g}$ states, and the two appear at nearly identical peak positions at around $-0.7 \mathrm{eV}$ (relative to the VBM). The relative positions of the valence $e_{g}$ and $t_{2 g}$ bands $(-0.7$ and $-2.3 \mathrm{eV})$ are similar to what is seen in QPscGW $(-0.5$ and $-2.2 \mathrm{eV}$, respectively). Similarly, in $\mathrm{NiO}$, there is little $e_{g}$ weight (peak around $-0.4 \mathrm{eV}$ ) near the VBM, and the first peak for $t_{2 g}$ is found to be around $-1.0 \mathrm{eV}$. Compared with QPscGW, our calculation suggests less weight for $e_{g}$ around the VBM, and the location of $t_{2 g}$ is similar to the QPscGW finding $(\sim-1.0 \mathrm{eV})$. Note that additional valence $e_{g}$ peaks in $\mathrm{NiO}$ are expected to lie deeper in the spectrum [13] and thus do not appear in Fig. 6. Quasiparticle weights at the CBM and VBM in both materials are large $(\sim 0.9)$.

The observed $s$ character of the CBM in $\mathrm{MnO}$ and $\mathrm{NiO}$ is also found in some earlier GGA-based GW calculations
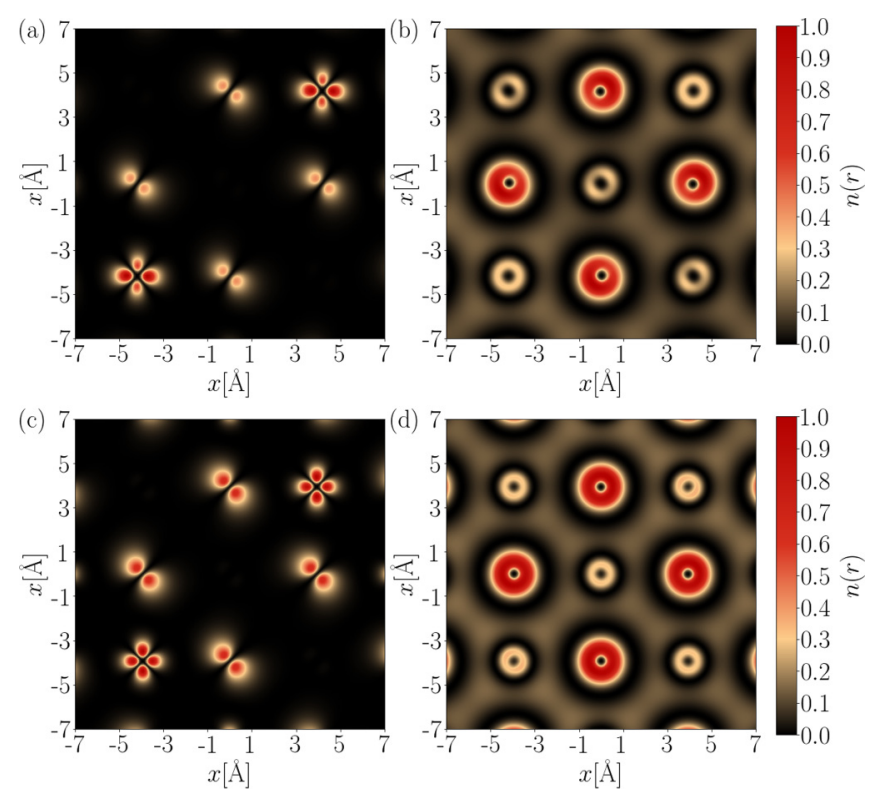

FIG. 8. Spatial density distribution of quasiparticle orbitals on the (100) surface for (a) MnO VBM, (b) CBM, (c) NiO VBM, and (d) CBM. For $\mathrm{MnO}$, we show the $x y$ plane where the projected ionization charge shows $e_{g}$ symmetry, and for $\mathrm{NiO}$, the quasiparticle orbitals are projected onto the $x z$ plane.

[14] but not others $[15,16]$. This feature was missed in early DMFT impurity model calculations where the Ni impurity was defined using only the $3 d$ shell [20-22], although it has been seen in more careful treatments in very recent DMFT calculations $[25,27,28]$. The orbital character of the CBM and VBM, including the $s$ character, can be visualized explicitly in real space by defining quasiparticle orbitals for the CBM/VBM excitation,

$$
\begin{aligned}
& \left|\psi_{k}^{-}\right\rangle=\sum_{i} r_{i k}\left|\phi_{i k}\right\rangle, \\
& \left|\psi_{k}^{+}\right\rangle=\sum_{a} r^{a k}\left|\phi_{a k}\right\rangle,
\end{aligned}
$$

where $\phi_{i k}$ and $\phi_{a k}$ are occupied and virtual mean-field orbitals with crystal momentum $k$. Real-space density plots of the quasiparticle orbitals at the VBM and CBM are shown in Fig. 8.

From the analysis above, both $\mathrm{MnO}$ and $\mathrm{NiO}$ appear as insulators of mixed charge-transfer/Mott character. However, this picture is not uniform across the Brillouin zone. In particular, when only the fundamental gap is examined, $\mathrm{NiO}$ is clearly a charge-transfer insulator, while $\mathrm{MnO}$ remains of mixed character. Thus the nature of the insulating state in these systems should be regarded as momentum dependent.

\section{CONCLUSION}

In conclusion, we have carried out a detailed study of the ground and excited states of $\mathrm{MnO}$ and $\mathrm{NiO}$ using coupled cluster theory. While the description of the spectrum is significantly improved over mean-field methods and quantitatively accurate at the level of $2 \times 2 \times 2$ supercells, the gaps in the thermodynamic limit remain somewhat overestimated, likely 
due to orbital relaxation effects and higher-order excitations. Unfortunately, we are not yet able to provide a quantitative estimate of the effect of triples due to the prohibitive cost. Nonetheless, coupled cluster offers interesting insights into the qualitative nature of the insulating state in these materials, allowing for a detailed analysis of the charge-transfer/Mottinsulating character, atomic character of the bands (which indicates the important participation of $s$ character states in the conduction band minima), and quasiparticle weights. Most intriguingly, our results show that the charge-transfer Mott nature of the insulating state should be considered to be a momentum-dependent quantity. Our work is a significant step towards the use of periodic coupled cluster methods to understand correlated electronic materials.

\section{ACKNOWLEDGMENTS}

We thank Z. Cui and T. Zhu for helpful discussions on spectrum analysis. G.K.-L. C. acknowledges support from Grant No. DE-SC0018140. Partial support for Y.G. was from
Grant No. DE-SC0019330. A.F.W. was supported by MURI Grant No. FA9550-18-1-0095. J.M.Y. acknowledges support from NSF Grant No. DGE-1745301. Y.G. and A.J.M. acknowledge the support of ONR under Grant No. N00014-18$1-2101$.

\section{APPENDIX: AMPLITUDE EQUATIONS}

Here we provide equations for both the ground state and IP/EA EOM CCSD starting from an unrestricted mean-field reference. For compactness, we use bold letters to represent a combined spin-orbital and $k$-point index, i.e., $\mathbf{i}=\left(i, k_{i}\right)$. Lowercase $i, j, a, b$ and uppercase $I, J, A, B$ are used for spin-up and spin-down orbitals, respectively. The primed sums indicate momentum conservation. The intermediates $W, F, \tilde{W}$, and $\tilde{F}$ that we use are consistent with those in Stanton et al. [40,41]. $P(\mathbf{a b})$ is the antisymmetry operator such that $[P(\mathbf{a b}) Z(\cdots a b \cdot \cdot)=Z(\cdots a b \cdots)-Z(\cdots b a \cdot \cdot \cdot)]$ for any variable $Z$.

The single-excitation amplitudes for the up-spin component are obtained from the following equations:

$$
\begin{aligned}
0= & f_{\mathbf{a i}}+{ }^{\prime} \sum_{\mathbf{m e}}^{\prime} t_{\mathbf{i m}}^{\mathbf{a e}} \tilde{F}_{\mathbf{m e}}+\sum_{\mathbf{M E}}^{\prime} t_{\mathbf{i M}}^{\mathbf{a} \mathbf{E}} \tilde{F}_{\mathbf{M E}}-\sum_{\mathbf{m n e}}^{\prime} t_{\mathbf{m n}}^{\mathbf{a e}}\langle\mathbf{m n} \mid \mathbf{i e}\rangle-\sum_{\mathbf{m N E}}^{\prime} t_{\mathbf{m N}}^{\mathbf{a E}}\langle\mathbf{m N} \mid \mathbf{i E}\rangle+\sum_{e}^{\prime} t_{\mathbf{i}}^{\mathbf{e}} \tilde{F}_{\mathbf{a e}}-\sum_{m}^{\prime} t_{\mathbf{m}}^{\mathbf{a}} \tilde{F}_{\mathbf{m i}}+\sum_{\mathbf{m e}}^{\prime} t_{\mathbf{m e}}\langle\mathbf{a m}|| \mathbf{i} \mathbf{e}\rangle \\
& +\sum_{\mathbf{M E}}^{\prime} t_{\mathbf{M}}^{\mathbf{E}}\langle\mathbf{a M} \mid \mathbf{i E}\rangle+\sum_{\mathbf{m e f}}^{\prime} t_{\mathbf{i m}}^{\mathbf{e f}}\langle\mathbf{a m} \mid \mathbf{e f}\rangle+\sum_{\mathbf{M e F}} t_{\mathbf{i M}}^{\mathbf{e F}}\langle\mathbf{a M} \mid \mathbf{e F}\rangle .
\end{aligned}
$$

Similarly, the equations for $t_{\mathbf{I}}^{\mathbf{A}}$ can be obtained by flipping the spin.

The doubles amplitude equations for $t_{\mathbf{i j}}^{\mathbf{a b}}$ are

$$
\begin{aligned}
0= & \langle\mathbf{a b} \| \mathbf{i j}\rangle+P(\mathbf{a b}) \sum_{e}^{\prime} t_{\mathbf{i j}}^{\mathbf{a e}}\left[\tilde{F}_{\mathbf{b e}}-\sum_{\mathbf{m}}^{\prime} \frac{1}{2} t_{\mathbf{m}}^{\mathbf{b}} \tilde{F}_{\mathbf{m e}}\right]-P(\mathbf{i j}) \sum_{\mathbf{m}}^{\prime} t_{\mathbf{i m}}^{\mathbf{a b}}\left[\tilde{F}_{\mathbf{m} \mathbf{j}}+\sum_{\mathbf{e}}^{\prime} \frac{1}{2} t_{\mathbf{j}}^{\mathbf{e}} \tilde{F}_{\mathbf{m e}}\right]+\sum_{\mathbf{m n}}^{\prime} \frac{1}{2} \tilde{W}_{\mathbf{m n i j}} \tau_{\mathbf{m n}}^{\mathbf{a b}} \\
& +\sum_{\mathbf{c d}}^{\prime} \frac{1}{2} \tilde{W}_{\mathbf{a b c d}} \tau_{\mathbf{i j}}^{\mathbf{c d}}+P(\mathbf{i j}) P(\mathbf{a b}) \sum_{\mathbf{m e}}^{\prime} t_{\mathbf{i}}^{\mathbf{e}} t_{\mathbf{m}}^{\mathbf{a}}\langle\mathbf{m b}|| \mathbf{j e}\rangle+P(\mathbf{i j}) P(\mathbf{a b})\left[\sum_{\mathbf{m} \mathbf{e}}^{\prime} t_{\mathbf{i m}}^{\mathbf{a e}} \tilde{W}_{\mathbf{m b e j}}+\sum_{\mathbf{M E}}^{\prime} t_{\mathbf{i M}}^{\mathbf{a} \mathbf{a}} \tilde{W}_{\mathbf{M b E j}}\right] \\
& +\sum_{\mathbf{e}}^{\prime} P(\mathbf{i j}) t_{\mathbf{i}}^{\mathbf{e}}\langle\mathbf{b a}|| \mathbf{j e}\rangle-\sum_{\mathbf{m}}^{\prime} P(\mathbf{a b}) t_{\mathbf{m}}^{\mathbf{a}}\langle\mathbf{m b} \| \mathbf{i j}\rangle .
\end{aligned}
$$

The equations for $t_{\mathbf{I J}}^{\mathbf{A B}}$ can be obtained by flipping the spin. The equations for $t_{\mathbf{i J}}^{\mathbf{a B}}$ are

$$
\begin{aligned}
& 0=\langle\mathbf{a B} \mid \mathbf{i} \mathbf{J}\rangle+\sum_{m N}^{\prime} \tilde{W}_{\mathbf{m N i J}} \tau_{\mathbf{m N}}^{\mathbf{a B}}+\sum_{\mathbf{c D}}^{\prime} \tilde{W}_{\mathbf{a B c D}} \tau_{\mathbf{i J}}^{\mathbf{c D}}+\sum_{\mathbf{E}}^{\prime} t_{\mathbf{i J}}^{\mathbf{a} E}\left[\tilde{F}_{\mathbf{B E}}-\sum_{\mathbf{M}}^{\prime} \frac{1}{2} t_{\mathbf{M}}^{\mathbf{B}} \tilde{F}_{\mathbf{B E}}\right]+\sum_{\mathbf{e}}^{\prime} t_{\mathbf{i} \mathbf{J}}^{\mathbf{e B}}\left[\tilde{F}_{\mathbf{a e}}-\sum_{\mathbf{m}}^{\prime} \frac{1}{2} t_{\mathbf{m}}^{\mathbf{b}} \tilde{F}_{\mathbf{b e}}\right] \\
& -\sum_{\mathbf{M}}^{\prime} t_{\mathbf{i M}}^{\mathbf{a B}}\left[\tilde{F}_{\mathbf{M J}}+\sum_{\mathbf{E}}^{\prime} \frac{1}{2} t_{\mathbf{J}}^{\mathbf{E}} \tilde{F}_{\mathbf{M E}}\right]-\sum_{\mathbf{m}}^{\prime} t_{\mathbf{m J}}^{\mathbf{a B}}\left[\tilde{F}_{\mathbf{m i}}+\sum_{\mathbf{e}}^{\prime} \frac{1}{2} t_{\mathbf{i}}^{\mathbf{e}} \tilde{F}_{\mathbf{m e}}\right]+\sum_{\mathbf{m e}}^{\prime} t_{\mathbf{i m}}^{\mathbf{a e}} \tilde{W}_{\mathbf{m B e J}}+\sum_{\mathbf{M E}}^{\prime} t_{\mathbf{i M}}^{\mathbf{a E}} \tilde{W}_{\mathbf{M B E J}}-\sum_{\mathbf{m e}}^{\prime} t_{\mathbf{i}}^{\mathbf{e}} t_{\mathbf{m}}^{\mathbf{a}}\langle\mathbf{e J} \mid \mathbf{m B}\rangle \\
& +\sum_{\mathbf{m e}}^{\prime} t_{\mathbf{m} J}^{\mathbf{e} \mathbf{B}} \tilde{W}_{\mathbf{m a e i}}+\sum_{\mathbf{M E}}^{\prime} t_{\mathbf{M J}}^{\mathbf{E B}} \tilde{W}_{\mathbf{M a E i}}-\sum_{\mathbf{M E}}^{\prime} t_{\mathbf{J}}^{\mathbf{E}} t_{\mathbf{M}}^{\mathbf{B}}\langle\mathbf{a M} \mid \mathbf{i E}\rangle+\sum_{\mathbf{M e}}^{\prime} t_{\mathbf{i M}}^{\mathbf{e B}} \tilde{W}_{\mathbf{M a e}}+\sum_{\mathbf{m E}}^{\prime} t_{\mathbf{m J}}^{\mathbf{a E}} \tilde{W}_{\mathbf{m B E i}}-\sum_{\mathbf{M e}}^{\prime} t_{\mathbf{i}}^{\mathbf{e}} t_{\mathbf{M}}^{\mathbf{B}}\langle\mathbf{M a} \mid \mathbf{J e}\rangle \\
& -\sum_{\mathbf{m E}}^{\prime} t_{\mathbf{J}}^{\mathbf{E}} t_{\mathbf{m}}^{\mathbf{a}}\langle\mathbf{m B} \mid \mathbf{i E}\rangle+\sum_{\mathbf{e}}^{\prime} t_{\mathbf{i}}^{\mathbf{e}}\langle\mathbf{B a} \mid \mathbf{J e}\rangle+\sum_{\mathbf{E}}^{\prime} t_{\mathbf{J}}^{\mathbf{E}}\langle\mathbf{a B} \mid \mathbf{i E}\rangle-\sum_{\mathbf{m}}^{\prime} t_{\mathbf{m}}^{\mathbf{a}}\langle\mathbf{i} \mathbf{J} \mid \mathbf{m B}\rangle-\sum_{\mathbf{M}}^{\prime} t_{\mathbf{M}}^{\mathbf{B}}\langle\mathbf{J i} \mid \mathbf{M a}\rangle .
\end{aligned}
$$

The remaining mixed-spin excitation amplitudes can be obtained via permutational symmetry and need not be computed explicitly,

$$
t_{\mathbf{i J}}^{\mathbf{a B}}=t_{\mathbf{J i}}^{\mathbf{B a}}=-t_{\mathbf{i J}}^{\mathbf{B a}}=-t_{\mathbf{J i}}^{\mathbf{a B}}
$$


The IP-EOM amplitude equations are given by

$$
\begin{aligned}
& (\bar{H} R)_{\mathbf{i}}=-\sum_{\mathbf{k}}^{\prime} F_{\mathbf{k i}} r_{\mathbf{k}}-\sum_{\mathbf{L D}}^{\prime} F_{\mathbf{L D}} r_{\mathbf{L i}}^{\mathbf{D}}-\sum_{\mathbf{l d}}^{\prime} F_{\mathbf{l d}} r_{\mathbf{l i}}^{\mathbf{d}}-\frac{1}{2} \sum_{\mathbf{k l d}}^{\prime} W_{\mathbf{k l i d}} r_{\mathbf{l k}}^{\mathbf{d}}-\sum_{\mathbf{k L D}}^{\prime} W_{\mathbf{k L i D}} r_{\mathbf{L k}}^{\mathbf{D}}, \\
& (\bar{H} R)_{\mathbf{J i}}^{\mathbf{B}}=\sum_{\mathbf{k}}^{\prime} W_{\mathbf{k B i J}} r_{\mathbf{k}}-\sum_{\mathbf{l}}^{\prime} r_{\mathbf{J l}}^{\mathbf{B}} F_{\mathbf{l i}}-\sum_{\mathbf{L}}^{\prime} r_{\mathbf{L i}}^{\mathbf{B}} F_{\mathbf{L J}}+\sum_{\mathbf{D}}^{\prime} r_{\mathbf{J i}}^{\mathbf{D}} F_{\mathbf{B D}}+\sum_{\mathbf{L D}}^{\prime} W_{\mathbf{L B D J}} r_{\mathbf{L i}}^{\mathbf{D}}+\sum_{\mathbf{l d}}^{\prime} W_{\mathbf{I B d J}} r_{\mathbf{l i}}^{\mathbf{d}}+\sum_{\mathbf{I D}}^{\prime} W_{\mathbf{I B D i}} r_{\mathbf{J I}}^{\mathbf{D}}+\sum_{\mathbf{K L}}^{\prime} W_{\mathbf{k L i j}} r_{\mathbf{L k}}^{\mathbf{B}} \\
& -\sum_{\mathbf{c}}^{\prime} t_{\mathbf{i J}}^{\mathbf{c B}}\left[\sum_{\mathbf{k L D}}^{\prime} W_{\mathbf{L k D c}} r_{\mathbf{L k}}^{\mathbf{D}}+\frac{1}{2} \sum_{\mathbf{k l d}}^{\prime} W_{\mathbf{l k d c}} r_{\mathbf{l k}}^{\mathbf{d}}\right] \text {, } \\
& (\bar{H} R)_{\mathbf{i j}}^{\mathbf{b}}=-\sum_{\mathbf{k}}^{\prime} W_{\mathbf{k b j i}} r_{\mathbf{k}}-\sum_{k_{l}}^{\prime} \sum_{\mathbf{l}} F_{\mathbf{l i}} r_{\mathbf{j} 1}^{\mathbf{b}}+\sum_{\mathbf{l}}^{\prime} F_{\mathbf{l j}} r_{\mathbf{i l}}^{\mathbf{b}}+P(\mathbf{i j})\left(\sum_{\mathbf{L D}}^{\prime} W_{\mathbf{L b D j}} r_{\mathbf{L i}}^{\mathbf{D}}+\sum_{\mathbf{l d}}^{\prime} W_{\mathbf{l b d j}} r_{\mathbf{l i}}^{\mathbf{d}}\right) \\
& -\sum_{\mathbf{c}}^{\prime} t_{\mathbf{i j}}^{\mathbf{c b}}\left[\sum_{\mathbf{k L D}}^{\prime} W_{\mathbf{L k D c}} r_{\mathbf{L k}}^{\mathbf{D}}+\frac{1}{2} \sum_{\mathbf{k l d}}^{\prime} W_{\mathbf{l k d c}} r_{\mathbf{l k}}^{\mathbf{d}}\right]+\sum_{\mathbf{d}}^{\prime} F_{\mathbf{b d}} r_{\mathbf{j i}}^{\mathbf{d}}+\frac{1}{2} \sum_{\mathbf{k l}}^{\prime} W_{\mathbf{k l i j}} r_{\mathbf{l k}}^{\mathbf{b}} \text {. }
\end{aligned}
$$

The EA-EOM amplitude equations are given by

$$
\begin{gathered}
(\bar{H} R)^{\mathbf{a}}=\sum_{\mathbf{c}}^{\prime} F_{\mathbf{a c}} r^{\mathbf{c}}+\sum_{\mathbf{L D}}^{\prime} F_{\mathbf{L D}} r_{\mathbf{L}}^{\mathbf{D a}}+\sum_{\mathbf{l d}}^{\prime} F_{\mathbf{l d}} r_{\mathbf{l}}^{\mathbf{d a}}+\sum_{\mathbf{c L D}}^{\prime} W_{\mathbf{a L c D}} r_{\mathbf{L}}^{\mathbf{D c}}+\frac{1}{2} \sum_{\mathbf{c l d}}^{\prime} W_{\mathbf{a l c d}} r_{\mathbf{l}}^{\mathbf{d c}}, \\
(\bar{H} R)_{\mathbf{J}}^{\mathbf{B a}}=\sum_{\mathbf{c}}^{\prime} W_{\mathbf{a B c J}} r^{\mathbf{c}}+\sum_{\mathbf{c}}^{\prime} r_{\mathbf{J}}^{\mathbf{B c}} F_{\mathbf{a c}}+\sum_{\mathbf{D}}^{\prime} r_{\mathbf{J}}^{\mathbf{D a}} F_{\mathbf{B D}}-\sum_{\mathbf{k}}^{\prime} t_{\mathbf{k J}}^{\mathbf{a B}}\left[\sum_{\mathbf{c D L}}^{\prime} W_{\mathbf{k L c D}} r_{\mathbf{L}}^{\mathbf{D c}}+\frac{1}{2} \sum_{\mathbf{c d l}}^{\prime} W_{\mathbf{k l c d}} r_{\mathbf{l}}^{\mathbf{d c}}\right] \\
+\sum_{\mathbf{L D}}^{\prime} W_{\mathbf{L B D J}} r_{\mathbf{L}}^{\mathbf{D a}}+\sum_{\mathbf{l d}}^{\prime} W_{\mathbf{l B d J}} r_{\mathbf{l}}^{\mathbf{d a}}+\sum_{\mathbf{c L}}^{\prime} W_{\mathbf{L a c j}} r_{\mathbf{L}}^{\mathbf{B c}}-\sum_{\mathbf{L}}^{\prime} r_{\mathbf{L}}^{\mathbf{B a}} F_{\mathbf{L J}}+\sum_{\mathbf{c D}}^{\prime} W_{\mathbf{a B c D}} r_{\mathbf{J}}^{\mathbf{D c}}, \\
(\bar{H} R)_{\mathbf{j}}^{\mathbf{b a}}=\sum_{\mathbf{c}}^{\prime} W_{\mathbf{a b c j}} r^{\mathbf{c}}+\sum_{\mathbf{c}}^{\prime} F_{\mathbf{a c}} r_{\mathbf{j}}^{\mathbf{b c}}+\sum_{\mathbf{d}}^{\prime} F_{\mathbf{b d}} r_{\mathbf{j}}^{\mathbf{d a}}-\sum_{\mathbf{l}}^{\prime} F_{\mathbf{l j}} r_{\mathbf{l}}^{\mathbf{b a}}+\sum_{\mathbf{c d}}^{\prime} \frac{1}{2} W_{\mathbf{a b c d}} r_{\mathbf{j}}^{\mathbf{d c}}-\sum_{\mathbf{L D}}^{\prime} W_{\mathbf{L a D j}} r_{\mathbf{L}}^{\mathbf{D b}} \\
-\sum_{\mathbf{k}}^{\prime} t_{\mathbf{k j}}^{\mathbf{a b}}\left[\frac{1}{2} \sum_{\mathbf{c d l}}^{\prime} W_{\mathbf{k l c d}} r_{\mathbf{l}}^{\mathbf{d c}}+\sum_{\mathbf{c D L}}^{\prime} W_{\mathbf{L k D c}} r_{\mathbf{L}}^{\mathbf{D c}}\right]+\sum_{\mathbf{l d}}^{\prime} W_{\mathbf{l b d j}} r_{\mathbf{l}}^{\mathbf{d a}}+\sum_{\mathbf{L D}}^{\prime} W_{\mathbf{L b D j}} r_{\mathbf{L}}^{\mathbf{D a}}-\sum_{\mathbf{l d}}^{\prime} W_{\mathbf{l a d j}} r_{\mathbf{l}}^{\mathbf{d b}} .
\end{gathered}
$$

[1] G. A. Sawatzky and J. W. Allen, Phys. Rev. Lett. 53, 2339 (1984).

[2] J. van Elp, R. H. Potze, H. Eskes, R. Berger, and G. A. Sawatzky, Phys. Rev. B 44, 1530 (1991).

[3] A. Fujimori, N. Kimizuka, T. Akahane, T. Chiba, S. Kimura, F. Minami, K. Siratori, M. Taniguchi, S. Ogawa, and S. Suga, Phys. Rev. B 42, 7580 (1990).

[4] N. F. Mott, Proc. Phys. Soc. A 62, 416 (1949).

[5] A. Fujimori, F. Minami, and S. Sugano, Phys. Rev. B 29, 5225 (1984).

[6] X. Wang, M. J. Han, L. de' Medici, H. Park, C. A. Marianetti, and A. J. Millis, Phys. Rev. B 86, 195136 (2012).

[7] K. Terakura, A. R. Williams, T. Oguchi, and J. Kübler, Phys. Rev. Lett. 52, 1830 (1984).

[8] M. Marsman, J. Paier, A. Stroppa, and G. Kresse, J. Phys.: Condens. Matter 20, 064201 (2008).

[9] T. Bredow and A. R. Gerson, Phys. Rev. B 61, 5194 (2000).

[10] C. Franchini, V. Bayer, R. Podloucky, J. Paier, and G. Kresse, Phys. Rev. B 72, 045132 (2005).

[11] F. Ma, W. Purwanto, S. Zhang, and H. Krakauer, Phys. Rev. Lett. 114, 226401 (2015).
[12] C. Mitra, J. T. Krogel, J. A. Santana, and F. A. Reboredo, J. Chem. Phys. 143, 164710 (2015).

[13] S. V. Faleev, M. van Schilfgaarde, and T. Kotani, Phys. Rev. Lett. 93, 126406 (2004).

[14] J.-L. Li, G.-M. Rignanese, and S. G. Louie, Phys. Rev. B 71, 193102 (2005).

[15] S. Massidda, A. Continenza, M. Posternak, and A. Baldereschi, Phys. Rev. B 55, 13494 (1997).

[16] F. Aryasetiawan and O. Gunnarsson, Phys. Rev. Lett. 74, 3221 (1995).

[17] V. I. Anisimov, J. Zaanen, and O. K. Andersen, Phys. Rev. B 44, 943 (1991).

[18] L. Wang, T. Maxisch, and G. Ceder, Phys. Rev. B 73, 195107 (2006).

[19] G. Trimarchi, Z. Wang, and A. Zunger, Phys. Rev. B 97, 035107 (2018).

[20] J. Kuneš, V. I. Anisimov, A. V. Lukoyanov, and D. Vollhardt, Phys. Rev. B 75, 165115 (2007).

[21] J. Kuneš, V. I. Anisimov, S. L. Skornyakov, A. V. Lukoyanov, and D. Vollhardt, Phys. Rev. Lett. 99, 156404 (2007). 
[22] X. Ren, I. Leonov, G. Keller, M. Kollar, I. Nekrasov, and D. Vollhardt, Phys. Rev. B 74, 195114 (2006).

[23] K. Byczuk, J. Kuneš, W. Hofstetter, and D. Vollhardt, Phys. Rev. Lett. 108, 087004 (2012).

[24] P. Thunström, I. Di Marco, and O. Eriksson, Phys. Rev. Lett. 109, 186401 (2012).

[25] S. Mandal, K. Haule, K. M. Rabe, and D. Vanderbilt, Phys. Rev. B 100, 245109 (2019).

[26] I. Leonov, L. Pourovskii, A. Georges, and I. A. Abrikosov, Phys. Rev. B 94, 155135 (2016).

[27] L. Zhang, P. Staar, A. Kozhevnikov, Y.-P. Wang, J. Trinastic, T. Schulthess, and H.-P. Cheng, Phys. Rev. B 100, 035104 (2019).

[28] T. Zhu, Z.-H. Cui, and G. K. Chan, J. Chem. Theory Comput. 16, 141 (2020).

[29] I. Shavitt and R. J. Bartlett, Many-Body Methods in Chemistry and Physics: MBPT and Coupled-Cluster Theory (Cambridge University Press, Cambridge, 2009).

[30] R. J. Bartlett and M. Musiał, Rev. Mod. Phys. 79, 291 (2007).

[31] J. Č́ížek, J. Chem. Phys. 45, 4256 (1966).

[32] J. McClain, Q. Sun, G. K.-L. Chan, and T. C. Berkelbach, J. Chem. Theory Comput. 13, 1209 (2017).

[33] T. Gruber, K. Liao, T. Tsatsoulis, F. Hummel, and A. Grüneis, Phys. Rev. X 8, 021043 (2018).

[34] H. J. Monkhorst, Int. J. Quantum Chem. 12, 421 (1977).

[35] A. I. Krylov, Annu. Rev. Phys. Chem. 59, 433 (2008).

[36] J. F. Stanton and R. J. Bartlett, J. Chem. Phys. 98, 7029 (1993).

[37] J. McClain, J. Lischner, T. Watson, D. A. Matthews, E. Ronca, S. G. Louie, T. C. Berkelbach, and G. K.-L. Chan, Phys. Rev. B 93, 235139 (2016).

[38] M. F. Lange and T. C. Berkelbach, J. Chem. Theory Comput. 14, 4224 (2018).

[39] M. van Schilfgaarde, T. Kotani, and S. Faleev, Phys. Rev. Lett. 96, 226402 (2006).

[40] J. F. Stanton, J. Gauss, J. D. Watts, and R. J. Bartlett, J. Chem. Phys. 94, 4334 (1991).

[41] J. Gauss and J. F. Stanton, J. Chem. Phys. 103, 3561 (1995).
[42] A. K. Cheetham and D. A. O. Hope, Phys. Rev. B 27, 6964 (1983).

[43] J. VandeVondele and J. Hutter, J. Chem. Phys. 127, 114105 (2007).

[44] J. VandeVondele, M. Krack, F. Mohamed, M. Parrinello, T. Chassaing, and J. Hutter, Comput. Phys. Commun. 167, 103 (2005).

[45] Q. Sun, T. C. Berkelbach, J. D. McClain, and G. K.-L. Chan, J. Chem. Phys. 147, 164119 (2017).

[46] C. Gros, Z. Phys. B 86, 359 (1992).

[47] C. Lin, F. H. Zong, and D. M. Ceperley, Phys. Rev. E 64, 016702 (2001).

[48] J. F. Stanton, Chem. Phys. Lett. 281, 130 (1997).

[49] G. Knizia, J. Chem. Theory Comput. 9, 4834 (2013).

[50] Z.-H. Cui, T. Zhu, and G. K. Chan, J. Chem. Theory Comput. 16, 119 (2020).

[51] Z.-X. Shen, R. S. List, D. S. Dessau, B. O. Wells, O. Jepsen, A. J. Arko, R. Barttlet, C.-K. Shih, F. Parmigiani, J. C. Huang, and P. A. P. Lindberg, Phys. Rev. B 44, 3604 (1991).

[52] Q. Sun, T. C. Berkelbach, N. S. Blunt, G. H. Booth, S. Guo, Z. Li, J. Liu, J. D. McClain, E. R. Sayfutyarova, S. Sharma et al., Wiley Interdiscip. Rev.: Comput. Mol. Sci. 8, e1340 (2018).

[53] W. Kohn and L. J. Sham, Phys. Rev. 140, A1133 (1965).

[54] J. P. Perdew, K. Burke, and M. Ernzerhof, Phys. Rev. Lett. 77, 3865 (1996).

[55] A. D. Becke, J. Chem. Phys. 98, 1372 (1993).

[56] B. Fender, A. Jacobson, and F. Wedgwood, J. Chem. Phys. 48, 990 (1968).

[57] V. Fernandez, C. Vettier, F. de Bergevin, C. Giles, and W. Neubeck, Phys. Rev. B 57, 7870 (1998).

[58] N. J. DeYonker, K. A. Peterson, G. Steyl, A. K. Wilson, and T. R. Cundari, J. Phys. Chem. A 111, 11269 (2007).

[59] D. R. Lide, CRC Handbook of Chemistry and Physics: A ReadyReference Book of Chemical and Physical Data (CRC Press, Boca Raton, FL, 1995). 